\title{
Collaborative Virtual Reality for Low-Latency Interaction
}

\author{
Carmine Elvezio $\quad$ Frank Ling Jen-Shuo Liu Steven Feiner \\ Dept. of Computer Science, Columbia University, New York, USA \\ \{ce2236, ffl2107, j15004, feiner\}@ columbia.edu
}

(a)

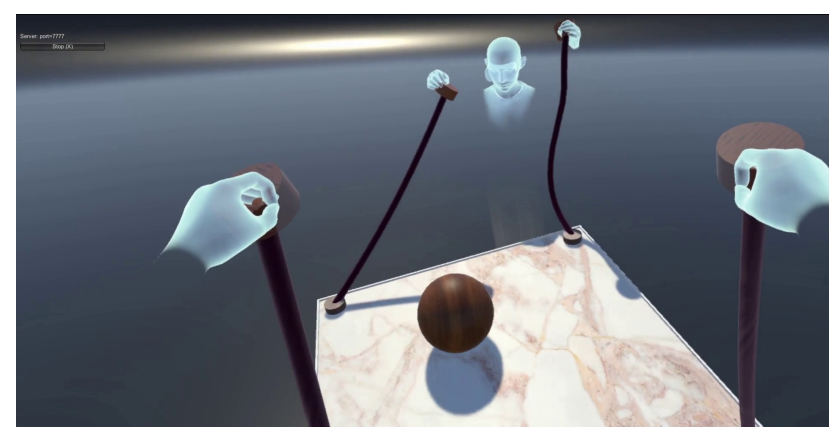

(b)

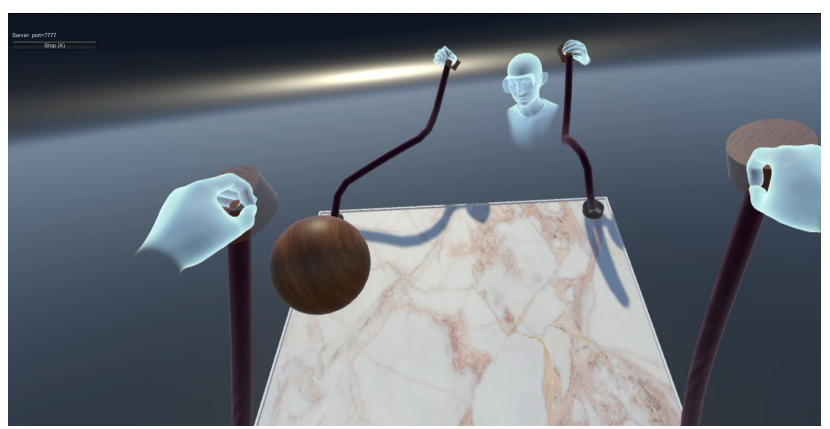

Figure 1. (a) Two users manipulate virtual board using four virtual ropes, one in each hand, to control virtual ball. (b) Users throw the ball upwards and try to catch it again. This experience requires low network latency for users to effectively coordinate their actions.

\begin{abstract}
In collaborative virtual environments, users must often perform tasks requiring coordinated action between multiple parties. Some cases are symmetric, in which users work together on equal footing, while others are asymmetric, in which one user may have more experience or capabilities than another (e.g., one may guide another in completing a task). We present a multi-user virtual reality system that supports interactions of both these types. Two collaborating users, whether co-located or remote, simultaneously manipulate the same virtual objects in a physics simulation, in tasks that require low latency networking to perform successfully. We are currently applying this approach to motor rehabilitation, in which a therapist and patient work together.
\end{abstract}

\section{CCS Concepts}

-Human-centered computing $\rightarrow$ Mixed / augmented reality; Collaborative interaction; Virtual reality; •Computing methodologies $\rightarrow$ Mixed / augmented reality; Virtual reality; -Software and its engineering $\rightarrow$ Interactive games;

\section{Author Keywords}

Virtual Reality; Collaboration; Games; Rehabilitation

\section{INTRODUCTION}

Collaborative virtual reality (VR) and augmented reality (AR) applications can allow multiple users to work together, si-

\footnotetext{
Permission to make digital or hard copies of part or all of this work for personal or classroom use is granted without fee provided that copies are not made or distributed for profit or commercial advantage and that copies bear this notice and the full citation on the first page. Copyrights for third-party components of this work must be honored. For all other uses, contact the owner/author(s).

UIST '18 Adjunct October 14-17, 2018, Berlin, Germany

(C) 2018 Copyright held by the owner/author(s).

ACM ISBN 978-1-4503-5949-8/18/10.
}

DOI: https : //doi.org/10.1145/3266037.3271643 multaneously, whether co-located or remote, in domains such as games, occupational training, and rehabilitation. Game engines, such as Unity and Unreal, simplify the creation of networked VR and AR applications. However, collaboration in these systems often proves to be complicated when it involves manipulation of shared virtual objects. Many collaborative applications allow individual users to manipulate separate objects when completing a shared task. In contrast, when a single object must be simultaneously manipulated by multiple users, it can become difficult to implement object control in a way that is understandable and satisfactory to all users.

We present a system that implements simultaneous shared virtual object manipulation through a set of parameterized elastically attached handles. This allows for a physicallymodeled representation of control of shared objects, where users can simultaneously exert control, in a way that is both comprehensible and consistent with the rules of virtual worlds following basic Newtonian physics.

\section{RELATED WORK}

Our research builds on two categories of related work: VR/AR for collaboration and for motor rehabilitation.

Much research has addressed the use of VR and AR for collaboration $[9,4,11]$, whether co-located or remote. In co-located collaboration, VR and AR can be used to show additional information to help facilite communication between users, (e.g., showing a virtual 3D object during class discussion [8]). On the other hand, in remote collaboration, VR and AR can help transmit information that is difficult to express verbally (e.g., maintenance and repair task assistance [10]).

VR and AR have also been used in motor rehabilitation [7], in which patients can practice specific body movements (e.g., 


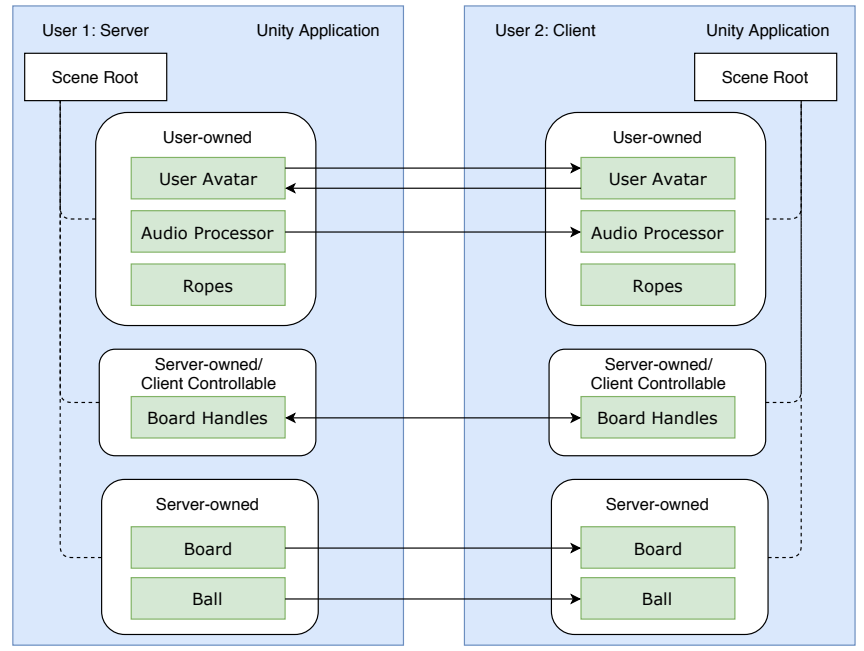

Figure 2. System architecture. Three types of objects are supported: User-owned objects are computed on each device, for that user. Serverowned/client-controllable objects are owned by server, but can be updated by any user on network. Server-owned objects are owned and updated by server, with updates sent authoritatively to all clients.

rehabilitation for stroke [5], chronic pain [12], and hemiparetic cerebral palsy [13]).

\section{IMPLEMENTATION}

Our system enables users to jointly manipulate a rigid body through the use of non-rigid control points connected to that rigid body. This allows the positions of users' hands to move outside the range of what is allowable using a non-deformable rigid body that they hold. Much previous work has focused on the efficient network synchronization of one or many virtual rigid bodies (e.g., [3]). This allows multiple users to interact with a synchronized set of many virtual objects. However, in these systems, a single object is often controlled exclusively by a single user at a time (e.g., when users alternate imparting force to a projectile in games such as ping pong or tennis). Simultaneous control by multiple users of a single object is not handled (except to prevent users from grabbing an object that is already held). In contrast, our system allows multiple users to simultaneously and continuously interact with a single object in an intuitive way.

In our demo, two attendees collaborate to control a virtual ball on a board. Two problems arise when considering the physical real-world analog to this scenario. First, without force feedback, we cannot prevent the users from moving their hands beyond the virtual constraints of the board. Second, we must make it possible for multiple users, operating on separate computers, to simultaneously control the same virtual objects.

To address both issues, we allow two users to control the board, each holding a pair of virtual handles connected to virtual ropes, each of which is connected to one of the four corners of the board. Our Unity implementation uses the Obi Rope asset [2]. Ropes make possible increased parametrization of the experience. By varying their length, users can finetune certain movements. For example, in a rehabilitation system, a therapist may want the patient to work on overhead movements. Longer ropes would require the patient to raise their arms higher to raise the board off the ground.

During a session, participants use Oculus Rift head-worn displays with Touch controllers. Each handle is gripped and controlled by a single Touch controller, allowing participants to manipulate the handles with six degrees of freedom. As the handles are moved in space, they pull on the ropes, which in turn move the board.

Similar to other systems that require physics transmission across a network, we employ a single device to simulate the physics, which are then propagated to the other devices throughout the network. While the standard approach to implementing this type of system using Unity involves the direct use of UNet, our system instead uses the open-source MercuryMessaging toolkit $[6,1]$, which simplifies networking and provides abstractions that support other networking protocols.

Each of the networked objects in the scene has a Mercury network node component, which allows it to communicate over the network. The board, ball, and handles are all instantiated by the server (Figure 2). But where the server authoritatively updates the simulation of the board and ball across all clients, the handles support bidirectional communication (the clients can update the handles). Using Mercury, all transformation and state updates are handled using the same protocol, so the programmer focuses purely on the directionality of the updates. In addition, using Mercury to handle transformation synchronization allows us to go beyond the $29 \mathrm{~Hz}$ limit imposed by the Unity NetworkTransform component.

We find that users can collaborate effectively in this task when round trip network latency is below $15 \mathrm{~ms}$ and collaboration improves further at latencies of 3-7 ms. Each scene element is transmitted using Mercury as fast as that element updates. (Note that the cosmetic visual delay between the other user's Oculus avatar hands and handles, seen in the video, is caused by a difference in avatar processing rate that affects only the other user's hand graphics, not the physics simulation.)

\section{CONCLUSIONS AND FUTURE WORK}

We have presented a system that facilitates efficient control of shared virtual objects in multi-user collaborative environments requiring low network latency. We have tested its performance for remote collaboration across an experimental $5 \mathrm{G}$ network and we have begun to work together with colleagues in motor rehabilitation to refine and test the system, initially in colocated interaction between therapists and patients.

This is only one approach to supporting this type of networked infrastructure. Other potential solutions include disengaging a user's grip on a handle when the hand and handle no longer occupy the same virtual space or having each user directly hold a board that is itself elastic. We will implement these other approaches and run a user study to compare them under different amounts of latency.

\section{ACKNOWLEDGMENTS}

This material is based on work supported in part by the National Science Foundation under Grant IIS-1514429 and by a generous gift from Verizon. 


\section{REFERENCES}

1. Mercury Messaging: An open-source framework to facilitate nonspatial communication in the Unity game engine.

https://github.com/ColumbiaCGUI/MercuryMessaging (Last accessed: August 10, 2018).

2. Obi Rope. http://obi.virtualmethodstudio.com/ (Last accessed: July 11, 2018).

3. Oculus Networking. https://developer.oculus.com/blog/ networked-physics-in-virtual-reality-networking-astack-of-cubes-with-unity-and-physx/ (Last accessed: July 11, 2018).

4. Robert Anderson, David Gallup, Jonathan T. Barron, Janne Kontkanen, Noah Snavely, Carlos Hernández, Sameer Agarwal, and Steven M. Seitz. 2016. Jump: Virtual Reality Video. ACM Trans. Graph. 35, 6, Article 198 (Nov. 2016), 13 pages. DOI :

http://dx.doi.org/10.1145/2980179.2980257

5. J. W. Burke, M. D. J. McNeill, D. K. Charles, P. J. Morrow, J. H. Crosbie, and S. M. McDonough. 2009. Optimising engagement for stroke rehabilitation using serious games. The Visual Computer 25, 12 (27 Aug 2009), 1085. DOI : http://dx.doi.org/10.1007/s00371-009-0387-4

6. Carmine Elvezio, Mengu Sukan, and Steven Feiner. 2018. Mercury: A Messaging Framework for Modular UI Components. In Proceedings of the 2018 CHI Conference on Human Factors in Computing Systems (CHI '18). ACM, New York, NY, USA, Article 588, 12 pages. DOI: http://dx.doi.org/10.1145/3173574.3174162

7. Maureen K. Holden. 2005. Virtual Environments for Motor Rehabilitation: Review. CyberPsychology \& Behavior 8, 3 (2005), 187-211. DOI : http://dx.doi.org/10.1089/cpb.2005.8.187 PMID: 15971970.

8. Hannes Kaufmann and Dieter Schmalstieg. 2002. Mathematics and Geometry Education with Collaborative Augmented Reality. In ACM SIGGRAPH 2002 Conference Abstracts and Applications (SIGGRAPH '02).
ACM, New York, NY, USA, 37-41. DOI : http://dx.doi.org/10.1145/1242073.1242086

9. J. Mortensen, V. Vinayagamoorthy, M. Slater, A. Steed, B. Lok, and M. C. Whitton. 2002. Collaboration in Tele-immersive Environments. In Proceedings of the Workshop on Virtual Environments 2002 (EGVE '02). Eurographics Association, Aire-la-Ville, Switzerland, Switzerland, 93-101.

http://dl.acm.org/citation.cfm?id=509709.509724

10. Ohan Oda, Carmine Elvezio, Mengu Sukan, Steven Feiner, and Barbara Tversky. 2015. Virtual Replicas for Remote Assistance in Virtual and Augmented Reality. In Proceedings of the 28th Annual ACM Symposium on User Interface Software 38; Technology (UIST '15). ACM, New York, NY, USA, 405-415. DOI: http://dx.doi.org/10.1145/2807442.2807497

11. Sergio Orts-Escolano, Christoph Rhemann, Sean Fanello, Wayne Chang, Adarsh Kowdle, Yury Degtyarev, David Kim, Philip L. Davidson, Sameh Khamis, Mingsong Dou, Vladimir Tankovich, Charles Loop, Qin Cai, Philip A. Chou, Sarah Mennicken, Julien Valentin, Vivek Pradeep, Shenlong Wang, Sing Bing Kang, Pushmeet Kohli, Yuliya Lutchyn, Cem Keskin, and Shahram Izadi. 2016. Holoportation: Virtual 3D Teleportation in Real-time. In Proceedings of the 29th Annual Symposium on User Interface Software and Technology (UIST '16). ACM, New York, NY, USA, 741-754. DOI : http://dx.doi.org/10.1145/2984511.2984517

12. Christian Schönauer, Thomas Pintaric, and Hannes Kaufmann. 2011. Full Body Interaction for Serious Games in Motor Rehabilitation. In Proceedings of the $2 \mathrm{Nd}$ Augmented Human International Conference (AH '11). ACM, New York, NY, USA, Article 4, 8 pages. DOI: http://dx.doi.org/10.1145/1959826.1959830

13. Sung H You, Sung Ho Jang, Yun-Hee Kim, Yong-Hyun Kwon, Irene Barrow, and Mark Hallett. 2005. Cortical reorganization induced by virtual reality therapy in a child with hemiparetic cerebral palsy. Developmental Medicine amp; Child Neurology 47, 9 (2005), 628-635. DOI : http://dx.doi.org/10.1017/S0012162205001234 